\title{
Revista Biomédica
}

https://revistabiomedica.mx

\section{Implicaciones causadas por la presencia y segregación de translocaciones robertsonianas. Su diagnóstico y efectos en la población}

\author{
René Emiliano Esquivel-Quezada ${ }^{1}$, Carla García-Morales ${ }^{1}$, Julieta Castillo-Cadena ${ }^{1,2}$ \\ ${ }^{1}$ Facultad de Ciencias, Universidad Autónoma del Estado de México. ${ }^{2}$ Clínica Multidisciplinaria de Salud, Universidad Autónoma \\ del Estado de México, Toluca, Estado de México.
}

\begin{abstract}
Implications caused by the presence and segregation of Robertsonian translocations. Its diagnosis and effects on the population.

Robertsonian translocations consist of the fusion of the acrocentric chromosome's large arms, they are frequent in humans, however, their presence may remain undiagnosed, giving only a hint of their existence when carriers have fertility problems, although this does not happen in all cases. Being aware of the presence of such translocations could be important for the treating doctors, geneticists and couples who want to start a family without knowing their condition as carriers with the risk of having a carrier or unbalanced offspring. A descriptive review was carried out with the aim of providing health professionals with information on the latest advances regarding Robertsonian translocations, as well as providing an accessible introduction to the subject. Information generated from 2014 to 2020 was collected from scientific data bases. We selected 47 articles from 254, and according to their content they were grouped into four categories: translocations and their effect on fertility, translocations as a cause of congenital malformations; the importance and usefulness on pre-implantation diagnosis and the effect of translocations from a statistical approach. Publications related to the detection of translocations in individuals with a family history of spontaneous abortions are more common than those with a different approach. Information related to robertsonian translocations considering other implications is rare. We highlight the importance of a wide diffusion about the identification and
\end{abstract}

Historial del artículo

Recibido: 15 jun 2021

Aceptado: 7 sep 2021

Disponible en línea: 1 ene 2022

\section{Palabras clave}

Translocación robertsoniana, fertilidad, cariotipo, abortos, aberraciones cromosómicas, malformaciones congénitas.

Keywords

Robertsonian translocation, fertility, karyotype, miscarriage, chromosomal aberration, congenital malformations.

Copyright (C) 2022 por autores y Revista Biomédica.

Este trabajo está licenciado bajo las atribuciones de la Creative Commons (CC BY).

http://creativecommons.org/licenses/by/4.0/

*Autor para correspondencia:

Julieta Castillo Cadena, Universidad Autónoma del Estado de México, Facultad de Ciencias/ Clínica Multidisciplinaria de Salud, Laboratorio de Genética. Jesús Carranza, Núm. 205, Col. Universidad, C.P. 50130, Toluca, Estado de México

E-mail: jcastilloc@uaemex.mx https://revistabiomedica.mx 
implications of robertsonian translocations for a correct management of the affected.

\section{RESUMEN}

Las translocaciones robertsonianas se producen por la fusión de los brazos largos de dos cromosomas acrocéntricos y son frecuentes en los seres humanos. Sin embargo, su presencia puede no ser detectada en el fenotipo. Sólo se sospechan cuando los portadores tienen problemas de fertilidad, aunque no sucede en todos los casos. El conocimiento de la presencia de dichas translocaciones es relevante para los médicos tratantes y parejas que desean una familia sin saber que son portadores. Ya que su descendencia puede heredar la translocación robertsoniana y ser portadora o tener desbalance cromosómico lo que genera un fenotipo clínico. Se realizó una revisión descriptiva de la literatura, con el objetivo de proporcionar a los profesionales de la salud la información sobre los últimos avances referentes a las translocaciones robertsonianas, así como dar una introducción accesible al tema. Se buscó información de 2014 a 2020, en bases de datos de artículos científicos. Se encontraron 254 referencias y seleccionaron 47. Se identificaron cuatro énfasis de estudio: las translocaciones y su efecto en la fertilidad, las translocaciones como causa de malformaciones congénitas, la importancia y utilidad del diagnóstico pre-implantación, y el efecto de las translocaciones en la población desde un enfoque estadístico. Los artículos publicados relacionados con la detección de estas translocaciones en individuos con un historial familiar de abortos espontáneos fueron los más numerosos. Se pone de manifiesto la necesidad de hacer una difusión más amplia sobre la identificación e implicaciones de las translocaciones robertsonianas, para el manejo oportuno de los afectados.

\section{INTRODUCCIÓN}

Las translocaciones cromosómicas son el intercambio de segmentos entre cromosomas homólogos y no homólogos, con un punto de ruptura en cada uno de ellos (1). En las translocaciones balanceadas o recíprocas no se pierde ni gana material genético, por lo que no tienen efecto en el fenotipo del portador. Sin embargo, en algunos casos en los puntos de ruptura se pueden alterar los genes o sus regiones reguladoras y dar lugar a fenotipos clínicos (2). Las translocaciones robertsonianas ocurren por la fusión de los brazos largos de dos cromosomas acrocéntricos (pares 13, 14, 15, 21 y 22) homólogos o no homólogos. La ruptura de los brazos cortos de ambos cromosomas puede dar lugar a un derivativo Dicéntrico o bien, si la ruptura sucede en el brazo corto de un cromosoma y otra en el brazo largo del otro acrocéntrico puede dar lugar a un derivativo monocéntrico. Por lo general, se pierde el material genético de los brazos cortos que consta de heterocromatina donde se encuentran los genes para la formación de los organizadores nucleolares (NOR'S), lo que hace a los portadores fenotípicamente normales, aun con 45 cromosomas (3). Sin embargo, a pesar del fenotipo normal de los portadores, la ovogénesis y la espermatogénesis están severamente afectadas, porque se pueden producir gametos con desequilibrio cromosómico, que originan abortos espontáneos, hijos que hereden la translocación robertsoniana o que presenten anomalías cromosómicas no balanceadas (4).

La fertilidad es la capacidad de reproducción de los seres vivos. La infertilidad ocurre cuando se logra un embarazo, pero es interrumpido involuntariamente en algún momento y sus causas pueden ser de diferente naturaleza, tales como ginecobstétricas, endocrinológicas, inmunológicas y genéticas. El diagnóstico de la infertilidad por cualquier causa debe tener una valoración completa por un equipo multidisciplinario (5).

El aborto espontáneo se define como la pérdida involuntaria de un embarazo durante las primeras 20 semanas. Durante el primer trimestre afecta del 10 al $15 \%$ de los embarazos clínicamente comprobados. Alrededor del $50 \%$ de estas pérdidas tienen como etiología anormalidades cromosómicas. Un historial de abortos recurrente puede indicar la presencia de rearreglos cromosómicos en uno de los progenitores. Desde punto de vista de la genética, es importante suponer la posibilidad de que algún miembro de la pareja sea portador de 
una translocación robertsoniana como causa de la infertilidad. Otro factor a considerar, son los parámetros anormales en el semen. En $40 \%$ de los casos de infertilidad masculina hay una base genética. Las anomalías cromosómicas estructurales que incluyen las translocaciones habitualmente cursan con oligozoospermia. Las translocaciones robertsonianas tienen una incidencia de $0.9 \%$ en los varones infértiles, cifra nueve veces mayor que en la población general (3). En los varones portadores de translocaciones robertsonianas, la cantidad de espermatozoides con carga genética desbalanceada oscila entre 3.4 y $40 \%$. Un espermiograma con resultados anormales debe considerar la realización de un cariotipo (6), que es una tecnología de diagnóstico genómico usada para detectar cambios estructurales grandes en los cromosomas tales como las translocaciones. También está indicado hacer microarreglos de polimorfismo de nucleótido único (SNP) el cual ofrece mayor resolución y permite detectar variaciones en el número de copias, como las que se encuentran en los puntos de ruptura de las translocaciones (7).

Otro efecto de las translocaciones robertsonianas en los hombres portadores se da en el núcleo de los espermatozoides, donde las regiones organizadoras nucleolares (sitio del cromosoma donde están los clusters de genes ribosómicos) se concentran más en la periferia de la parte media del núcleo a diferencia de los espermatozoides control, donde se localizan más dispersos hacia el área interna (8). En hombres portadores de éstas y que exhiben efecto intercromosómico (fenómeno provocado por la presencia de cromosomas translocados en el que hay interferencia en la segregación de otros cromosomas no translocados), se hizo hibridación fluorescente in situ en muestras de semen con el objetivo de evaluar si hay relación entre anormalidades cromosómicas numéricas y ciertos modos de segregación. Se concluyó que espermatozoides numéricamente anormales contienen principalmente productos de segregación desbalanceada (9).

Para comprender el mecanismoporel cual se forman embriones cromosómicamente desbalanceados, hay que recordar cómo se comportan los cromosomas con una translocación robertsoniana durante la meiosis para la formación de los gametos. La profase 1, es la fase más larga de la meiosis 1 y es donde los cromosomas se hacen visibles, ocurre el intercambio de material genético entre cromosomas homólogos, desaparece el nucléolo, se forma el huso meiótico y la membrana nuclear desaparece. Está fase se divide en leptoteno, zigoteno, paquiteno, diploteno y diacinesis. En la de paquiteno, se da el entrecruzamiento. En el caso de translocaciones, se forma la llamada cruz de paquiteno (un trivalente) lo que favorece que los cromosomas homólogos puedan intercambiar información entre ellos, como se muestra en la figura 1, la segregación después del intercambio puede ser de cuatro formas: a) segregación alterna, b) adyacente $1, \mathrm{c}$ ) adyacente 2 y d) segregación 3:1. Se generan 8 gametos diferentes. La forma alterna es la única que origina gametos normales o portadores balanceados que conducen a la formación de cigotos viables. Las demás segregaciones dan lugar a gametos desbalanceados $\mathrm{y}$ en consecuencia embriones anormales con desbalance cromosómico (10).

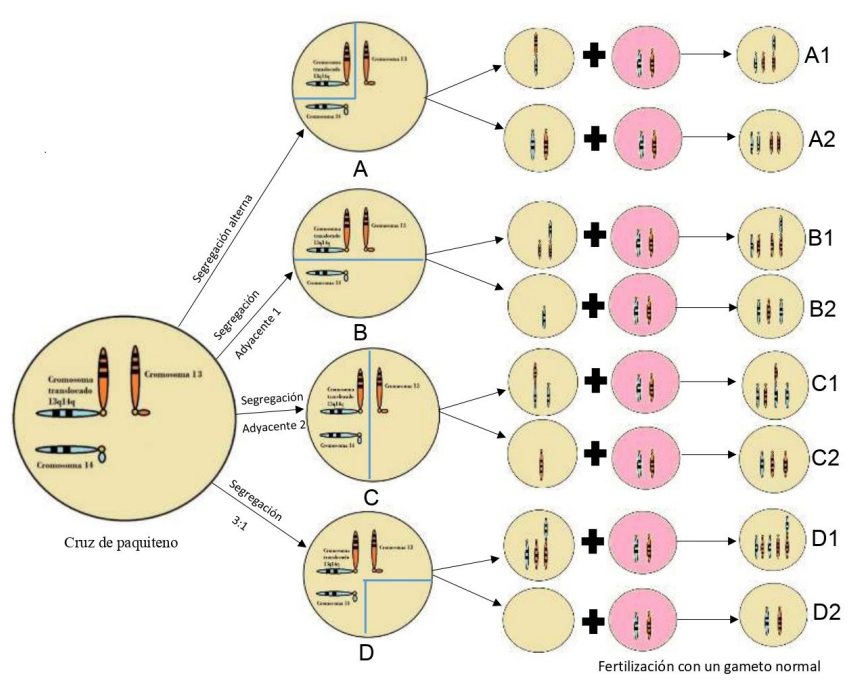

Figura 1. Diagrama que ejemplifica la cruz de paquiteno en un individuo con translocación robertsoniana en los cromosomas 13 y 14 , con sus posibles segregaciones y productos resultantes en caso de participar en una fertilización con un gameto normal. Elaboración personal. A. Al separarse los cromosomas de manera alterna, los dos cromosomas normales migran a un gameto, mientras que el cromosoma translocado migrará al otro. B. Cuando la segregación es horizontal, quedará un gameto con 
el cromosoma 13 más el translocado y al otro gameto solamente con el cromosoma 14. C. Cuando la segregación es vertical, un gameto constará del cromosoma 14 más el translocado y el otro gameto solamente con el cromosoma 13 presente. D. Cuando todo el material genético segrega a un gameto, el otro gameto carecerá de estos cromosomas. A1. Portador de la translocación balanceada, pero tendrá un fenotipo normal. A2. Producto con dotación cromosómica completa, fenotípica y cromosómicamente normal. B1. Trisomía del cromosoma 13 o síndrome de Patau. B2. Monosomía del cromosoma 13. C1. Trisomía del cromosoma 14. C2. Monosomía del cromosoma 14. D1. Producto inviable con presencia de tres cromosomas 13 y 14, dos de ellos translocados. D2. Producto inviable con ausencia de un cromosoma 13 y un 14 . De B2 a D2 se traducirá en un aborto espontáneo.

Las translocaciones robertsonianas tienen una frecuencia relativamente alta, 1 en 1,000 recién nacidos en la población y pueden permanecer sin diagnóstico durante toda la vida de los portadores (4). Estos individuos corren el riesgo de tener hijos portadores y perpetuar su presencia en la población o con malformaciones congénitas. En este caso, solo serán compatibles con la vida las trisomías regulares y parciales 21, 13 y las duplicaciones de los cromosomas 14 y 15, de ahí la importancia de su detección temprana. Este aspecto es poco considerado en el diagnóstico de la infertilidad, por tal motivo, esta revisión tuvo como objetivo compendiar la información existente sobre su diagnóstico, presencia en la población y las medidas que se han tomado para prevenir sus consecuencias, así como ofrecer una introducción accesible al tema.

\section{MATERIAL Y MÉTODO.}

Se realizó la revisión de la literatura enfocada en las investigaciones de 2014 a 2020. Se usaron las bases de datos Redalyc, Scielo, PubMed y el buscador "Google académico" para la recopilación de publicaciones sobre las translocaciones robertsonianas, su diagnóstico e implicaciones en la reproducción. Primero se realizó la búsqueda con "Robertsonian translocation", en PubMed se encontraron 254 resultados. Posteriormente se identificó en los títulos, resúmenes y objetivos la constante aparición de "case report" por lo que se incluyó el término en la búsqueda. Los resultados se agruparon entre los que implicaban abortos espontáneos recurrentes y malformaciones congénitas. También se identificó la aparición repetida de "Preimplantation genetic diagnosis" por lo que se incluyó también el término en la búsqueda y se encontraron artículos con un enfoque estadístico al incluir el término "study". Se repitió el proceso con el buscador académico de Google y Redalyc, donde se obtuvieron artículos realizados en Latinoamérica. Con el fin de incluir fuentes que proporcionen datos estadísticos en México se consideraron dos más, aunque que estaban fuera del periodo establecido. Finalmente, de acuerdo con los criterios de selección se realizó la revisión con 47 artículos.

\section{RESULTADOS}

La información se agrupó de acuerdo con el contenido en cuatro grandes enfoques identificados. El primero y más extenso fue cómo las translocaciones robertsonianas afectan la fertilidad de los que la poseen; el segundo, considera a las translocaciones como causa de malformaciones congénitas; el tercero resalta la importancia del diagnóstico pre-implantación y por último, el enfoque estadístico que analiza la frecuencia de estas alteraciones cromosómicas y su relación con otros factores de estudio.

\section{Las translocaciones cromosómicas y la fertilidad}

La frecuencia de las translocaciones robertsonianas es de un caso por cada mil recién nacidos (4) y entre estas, la que se da entre los cromosomas 13 y 14 representa el $75 \%$ de los casos (10). Las parejas que han presentado abortos espontáneos recurrentes tienen mayor riesgo de una aberración cromosómica estructural en uno de los progenitores, la incidencia de éstas en la población general es $0.7 \%$ y aumenta a $2.2 \%$ después de un aborto espontáneo, a $4.8 \%$ después de dos y a $5.2 \%$ después de tres. Dentro de las aberraciones cromosómicas estructurales, las más comunes son balanceadas, $61 \%$; seguidas por las translocaciones robertsonianas en un $16 \%$ (12).

En la comunidad de Cienfuegos, Cuba, se hizo un estudio retrospectivo de 2006 a 2016, de fetos 
diagnosticados con translocación balanceada o no. Se concentraron en 10 familias portadoras, 25 individuos en total. Se encontraron 17 casos de aberraciones estructurales, 13 de las cuales fueron translocaciones. Cuatro familias presentaron translocación robertsoniana entre los cromosomas 13 y 14 y una entre los cromosomas 14 y 21 o sea la mitad de las familias, con 13 portadores sanos y uno enfermo. En algunos casos, los familiares indicaron que los abortos espontáneos eran frecuentes en su familia. En estos, los autores recomiendan el estudio citogenético para toda la familia, asesoramiento genético y el seguimiento de futuros embarazos para la detección temprana de fetos afectados (2). Un estudio parecido se llevó a cabo en la Sección de Citogenética del Laboratorio Clínico de la Clínica Alemana de Santiago, en Chile de 1991 hasta 2013. Se realizaron 2,416 estudios citogenéticos en restos de abortos. Se encontraron anomalías cromosómicas en 956, de los cuales, 49 muestras tuvieron alteraciones estructurales, 15 translocaciones robertsonianas y ocho translocaciones no robertsonianas (13). Gran parte de las investigaciones encontradas, se enfocan en la fertilidad, la mayoría son casos de mujeres con historial de abortos espontáneos recurrentes. En la tabla 1, se muestra el detalle de los estudios encontrados. Se enfatiza la necesidad de análisis citogenéticos en parejas con infertilidad primaria (incapaz de lograr un embarazo después de un año de relaciones sexuales sin preservativo) y abortos espontáneos frecuentes. Antes de llevar a cabo cualquier técnica de reproducción asistida, se recomienda diagnóstico genético pre-implantación o prenatal en parejas portadoras de translocación robertsoniana (14).

Tabla 1. Reportes de casos de translocaciones con abortos espontáneos como antecedente

\begin{tabular}{llll}
\hline Autores y país & Antecedentes & Resultados & Observaciones o comentarios \\
\hline Torriani G, et al. & Se hizo cariotipo en el líquido & Resultado 45,XX,der(15;22)(q10;q10) Se continuó el embarazo y la niña \\
$2014(1)$. & amniótico de una gestante & mat,(5;17)(q31.3;q25) de novo. & nació sin manifestaciones clínicas, \\
Cuba & de 39 años debido a su edad & Translocación robertsoniana entre & pero permanece en seguimiento \\
& avanzada & los cromosomas 15q y 22q y una & clínico sistemático. \\
& & balanceada no robertsoniana entre & \\
& & los cromosomas 5 y 17 de novo en el \\
& feto. El cariotipo de la madre fue: 45,
\end{tabular}

Ozen F. et al. Se refirió a una pareja con 2014 (15). Turquía un historial de 3 abortos espontáneos a una clínica de investigación citogenética.

Vikraman SN, et Un aborto espontáneo a las al. 2015 (16). 6 semanas de gestación y India un niño de 10 meses con sospecha de Síndrome de Down.
El cariotipo dio como resultado 46, Se considera que la segregación XX en la mujer y 45, XYder(13q;14q) desbalanceada en los gametos es en el hombre. la causa de los abortos. En las familias de ambos integrantes de la pareja no se han reportado casos de abortos espontáneos

Resultado 46, XY,-21,+t (21q;21q), en el niño, su padre resultó normal y su madre $45, \mathrm{XX}$,$21,+21$ der21,t(21q;21q).
La madre solo tiene gametos con disomía 21 o nulisómicos, como consecuencia durante la fertilización el producto puede tener monosomía 21 (incompatible con la vida) o trisomía 21. 
Guapi VH, et al. Mujer de 31 años, con 6 2018 (4).

Ecuador

Kar B. et al 2018

(14).

India

Alhalabi N, et al.

2018 (17).

Irán

Ahmadi-

Shadmehri A, et

al. 2020 (18).

Irán

Poornima S, et al. 2020 (19).

Irán abortos espontáneos en el

primer trimestre de embarazo. espontáneos frecuentes embarazo. espontáneos
Translocación robertsoniana entre los cromosomas $13 \mathrm{q}$ y $15 \mathrm{q}$.

Se manifiesta la necesidad de cariotipar a los productos de los siguientes embarazos, sin dejar de lado a otros familiares antes de ofrecer asesoramiento genético

En ambos casos el varón era portador de la translocación robertsoniana, con cariotipo 45,XY,der(13;14)(q10;q10).

Ambos hombres también fueron diagnosticados con oligoastenoteratozoospermia.

Indica un origen de novo. al no encontrarse la translocación en otros miembros de la familia

Se encontró en la mujer una translocación robertsoniana durante el primer trimestre del 45,XX,der(22)(q10;q10). En el esposo un cariotipo normal al igual que otros miembros de la familia de la mujer.

Historial repetido de 4 abortos Translocación robertsoniana 45,XY,der(15;15)(10q;10q) en el progenitor masculino.

Translocation robertsoniana entre los cromosomas 13 y 14 y una translocación balanceada entre los cromosomas 4 y 7 en la madre $45 X X, t(4 ; 7)(\mathrm{q} 27 ; \mathrm{p} 22) \operatorname{der}(13 ; 14)$ (q q10;q10) y su hijo $45 \mathrm{XY}, \mathrm{t}(4 ; 7)$ (q27;p22)der(13;14) (q10;q10). El cariotipo del padre fue normal.
Historial de 3 abortos espontáneos en el primer trimestre de embarazo.

\section{Primer caso que involucra esta} translocación reportada en Irán.

Pérdidas recurrentes del embarazo por segregación anormal de la translocación balanceada.
Translocaciones como causa de malformaciones congénitas

Cuando se presentan malformaciones congénitas se recomienda un cariotipo con el fin de establecer el diagnóstico e identificar alguna anomalía cromosómica en los progenitores. Se encontraron 12 reportes de caso, que destacan la importancia de la realización del cariotipo para un asesoramiento genético oportuno (Tabla 2). 


\section{Esquivel-Quezada et al}

Tabla 2. Reportes de casos de portadores de translocaciones robertsonianas con antecedente de descendencia con malformaciones congénitas

\begin{tabular}{|c|c|c|c|}
\hline Autores & Antecedentes & Resultados & $\begin{array}{l}\text { Comentarios o Síndrome } \\
\text { diagnosticado. }\end{array}$ \\
\hline $\begin{array}{l}\text { Kusre G, } \\
\text { et al. } 2015 \\
\text { (20). } \\
\text { India }\end{array}$ & $\begin{array}{l}\text { Niña de } 10 \text { años con retraso mental y } \\
\text { defecto del tabique ventricular en el } \\
\text { corazón, músculos flácidos, retraso } \\
\text { en el crecimiento para la edad, ojos } \\
\text { oblicuos, cara y puente nasal planos, } \\
\text { habla inelegible y dificultad para } \\
\text { realizar sus actividades cotidianas. }\end{array}$ & $\begin{array}{l}\text { Paciente con cariotipo } \\
46, \mathrm{XX},+21, \text { der }(21 ; 21) \\
\text { (q10; } 10) \text {. Padres de cariotipo } \\
\text { normal, pero con un grado } 3 \text { de } \\
\text { consanguinidad. }\end{array}$ & $\begin{array}{l}\text { Síndrome de Down por } \\
\text { translocación robertsoniana de } \\
\text { novo }\end{array}$ \\
\hline $\begin{array}{l}\text { Kolgeci S, } \\
\text { et al. } 2015 \\
(21) . \\
\text { Kosovo }\end{array}$ & $\begin{array}{l}\text { Se realizó diagnóstico citogenético a } \\
\text { una pareja con un historial de abortos } \\
\text { espontáneos y descendencia con } \\
\text { síndrome de Down. }\end{array}$ & $\begin{array}{l}\text { El cariotipo de la madre es } 45, \\
\text { XX,der(14;21)(q10; q10), el del } \\
\text { padre es normal, } 46, X Y \text {. }\end{array}$ & $\begin{array}{l}\text { La madre ha tenido } 8 \text { embarazos } \\
\text { en total, dos de ellos resultaron en } \\
\text { hijos fenotípicamente normales, } \\
\text { un embarazo fue de gemelos, uno } \\
\text { de ellos fenotípicamente normal y } \\
\text { otro con síndrome de Down, dos } \\
\text { embarazos fueron interrumpidos } \\
\text { al detectarse síndrome de Down } \\
\text { en el feto y el último seguía en } \\
\text { curso al momento de realizado el } \\
\text { reporte de caso. }\end{array}$ \\
\hline
\end{tabular}

Jaiswal S, Caso 1: Padre e hijo ambos

et al. 2016 presentaban paladar submucoso

(22).

India

Cho $\mathrm{CH}$,

et al. 2016

(23).

Corea hendido y el hijo tenía trisomía

21. Caso 2: Madre e hija con translocación. Ambas hijas (la mencionada en el caso y su hermana mayor) presentan retraso global del desarrollo.

Fenotipo con hipotonía generalizada, dificultad respiratoria, labios superiores en forma de tienda, hiporreflexia y arteria umbilical única.
En el caso uno, cariotipo del padre y el hijo respectivamente fue: $45, X Y \operatorname{der}(13 ; 14)(q 10 ; q 10)$ y 46,XY,der(13;14)(q10;q10),+21. En el caso dos, madre e hija presentan

cariotipo: 45,XX, der(13;14) (q10;q10).

Translocación robertsoniana 45,XX,der(15;22) (q10;q10). En el segundo hijo de una pareja coreana.
Se presenta la relación fenotipocariotipo de la translocación robertsoniana der(13;14) en dos casos familiares. Se observó que las características clínicas varían con el punto de ruptura durante la translocación.

Refieren que las translocaciones que involucran a estos cromosomas representan el $0.6 \%$ de todos los casos. Se encontró deleción p15.11.2 lo que podría explicar las características clínicas presentes a pesar de ser una translocación robertsoniana.

Cariotipo 46,XY, +21, der(21;21) Síndrome de Down por (q10;q10). Padres con cariotipo normal. novo

Síndrome $\mathrm{X}$ con cariotipo 45,XY,der(13; 22)(q10; q10). El cariotipo de los padres no lo menciona
Es el primer caso reportado en que un paciente presenta a la vez síndrome $X$ frágil y una translocación robertsoniana. 
Malekpour

$\mathrm{N}$, et al.

2017 (26).

Irán
Historial de 4 abortos espontáneos y un hijo sano.

Desordenes esqueléticos en una niña.
Sha J, Se detectó en un análisis estructural et al. 2017 (27). Irán

Nikfar A, et al. 2019

(28).

Irán

Gumus E, $2019(21)$

Irán

Paciente 1: mosaico de síndrome de Paciente 2: Síndrome de Down. fetal malformación cardiaca fetal con ventrículo único, riñón poliquístico fetal y paladar hendido.

Deficiencia mental y discapacidad física.
En la madre se encontró una doble translocación robertsoniana $(44, \mathrm{XX}, \operatorname{der}(13 ; 14)$ (q10;q10)x2, es decir tenía dos veces los mismos cromosomas translocados. El infante solo presentaba una translocación robertsoniana entre los mismos cromosomas 45,XX,der(13q; $14 q)$, el padre era normal.

Se realizó cariotipo al feto, el resultado 46,XN,+13, der(13;14). El cariotipo de la madre fue $45, \mathrm{XX}, \operatorname{der}(13 ; 14)$ y el del padre $46, X Y$.

Cariotipo 46,XX, der(21;21) (q10;q10),+21, en el niño. El cariotipo de los padres fue normal.

El cariotipo del paciente uno fue 45,X[41]/47,XX,+21[59]. Los cariotipos de sus padres fueron normales.

El cariotipo del paciente dos es 46,XY,der(13;14)(q10;q10),+21. El cariotipo de su padre fue normal y el de su madre $45, \mathrm{XX}$, $\operatorname{der}(13 ; 14)(q 10 ; q 10)$.

Farzaneh
$\mathrm{MR}$, et al. 2019 (22).

Irán

$\mathrm{Xu} \mathrm{HH}$, et al. 2020 (19). China

Wang $\mathrm{X}$, et al. 2020 (29). Suiza Ambos con dismorfias faciales menores.

Síndrome de Turner

Síndrome de Down (previamente negativo en la prueba prenatal).

Infante masculino con dismorfia facial, tórax pequeño en forma de campana y hernia umbilical.
Se encontró cariotipo 44,X,der(13;14)(q10;q10). El cariotipo del padre 45,XY,

Cariotipo 46,XY, der(21;21)

(q10;q10),+21.

Sus padres resultaron normales

Se realizó cariotipo y se identificó una translocación robertsoniana $45, \mathrm{XY}$,der $(13 ; 14)$ (q10;q10) en el paciente y en su padre. Después se confirmó disomía uniparental 14. $\operatorname{der}(13 q ; 14 q)$.
Los desórdenes de la niña no son de causa cromosómica.

La descendencia de las mujeres $44, X X, \operatorname{der}(13 q ; 14 q) \times 2$ son portadores obligados de esta translocación

Se detectó síndrome de Patau en el feto, a causa de una translocación robertsoniana entre los cromosomas 13 y 14 heredada de la madre.

Síndrome de Down por translocación robertsoniana de novo

Las dismorfias faciales del paciente 2 son efecto de la trisomía 21.

Síndrome de Turner y translocación robertsoniana entre los cromosomas 13 y 14 heredada del padre

Síndrome de Down por translocación robertsoniana, de novo

Síndrome de Kagami-Okata, es el segundo caso en un varón reportado de manera oficial. 


\section{Esquivel-Quezada et al}

Alrededor de un $3 \%$ de los casos de síndrome de Down ocurren debido a una translocación robertsoniana, la más común entre los cromosomas 14 y 21 (2). Ésta se puede dar de manera espontánea o sea de novo o familiar, lo que implica que uno de los progenitores es portador de la translocación. Si tiene un origen materno la tasa de recurrencia de tener descendencia desbalanceada con trisomía es más alta comparada con las de origen paterno (33). Dentro de los casos de síndrome de Down causados por una translocación robertsoniana, el $25 \%$ es de origen familiar y $75 \%$ de origen de novo (34). En México, se hizo un estudio con el fin de identificar en pacientes con trisomía 21 que atendieron en el Hospital Infantil de México "Federico Gómez", el tipo y frecuencia de la aberración citogenética y evaluar el efecto de la edad de la madre. De 1921 casos identificados, $92(4.79 \%)$ se debían a una translocación robertsoniana (35).

Otro síndrome que puede ser causado por una translocación robertsoniana es el síndrome de Patau, el cual es la tercera trisomía autosómica más común, con una prevalencia de uno en 10,000-20,000 nacidos vivos. Es causado por una copia extra del cromosoma 13. Sus principales manifestaciones clínicas son; retraso en el desarrollo del sistema nervioso central, bajo peso al nacer, riñón poliquístico, severo retraso mental, cabeza pequeña y malformación cardiovascular. Se estima que, $50 \%$ de los niños que lo tienen mueren en un mes y $90 \%$ en un año. La translocación robertsoniana que más frecuentemente lo causa es entre los cromosomas, 13 y 14 la cual representa el $75 \%$ del total de translocaciones robertsonianas (27).

\section{La importancia del diagnóstico pre-implantación}

El diagnóstico genético pre-implantación es un estudio de DNA en tejido embrionario obtenido a partir de fertilización in vitro. Ofrece una oportunidad a las parejas portadoras de translocación robertsoniana de distinguir entre embriones normales y desbalanceados cromosómicamente, lo que aumenta las posibilidades de un embarazo exitoso (36).
Un reporte de caso de un portador de translocación robertsoniana con cariotipo 45,XY,der(21;21) (q10;q10), tuvo descendencia, una niña con un cariotipo mosaico 46,XX,[86]/46,XX, $\operatorname{der}(21 ; 21)$ [14], y sostiene que para los portadores de translocaciones robertsonianas homólogas, las tecnologías de asistencia para la reproducción como el uso de gametos de un donante y el diagnóstico genético pre-implantación pueden tener valor clínico (37).

El diagnóstico genético pre-implantación se usó en un análisis retrospectivo en portadores de translocación robertsoniana. Se compararon datos de embriones portadores de translocaciones robertsonianas en los días 3 y 5/6 de desarrollo en busca de un diferente comportamiento en los patrones de segregación. Se demostró un aumento de embriones genéticamente balanceados o normales en los días 5/6, lo que sugiere un proceso de selección natural entre los días 1-3 (10).

El diagnóstico genético pre-implantación permite la selección de embriones con equilibrio cromosómico, pero no permite detectar si un embrión es portador de una translocación, por lo que se ha empleado otra técnica llamada "Mapping Allele with Resolved Carrier Status", procedimiento que se ha comprobado como un método eficiente en la selección de embriones normales (38). Otra técnica descrita con este propósito es la del microarreglo de $\mathrm{CGH}$, que implica desnaturalización del DNA de la muestra y el uso de un referente etiquetado con fluoróforos e hibridación con una sonda. Aunque se trató de la primera aplicación clínica, no tuvo resultados concluyentes (39). Otra técnica complementaria al diagnóstico genético preimplantaciónes lasecuenciacióndenuevageneración, la cual provee nuevas posibilidades de diagnóstico y nuevos parámetros de evaluación, por ejemplo, la visualización de aneuploidías. Al usar estas dos técnicas se logró un embarazo exitoso en una pareja portadora de una translocación robertsoniana, la cual sufría de infertilidad primaria y en el 2009 le fue diagnosticado al varón oligospermia severa. Antes de lograr el embarazo exitoso, se intentó primero el uso de diagnóstico genético pre-implantación e 
hibridación fluorescente in situ lo que resultó en un aborto espontáneo en la semana ocho (40).

\section{Implicaciones de las translocaciones robertsonianas en la población desde un enfoque estadístico}

Existen pocos estudios con enfoque estadístico. En Gran Bretaña se realizó un estudio de cohorte. Se siguió a 1987 portadores de translocaciones robertsonianas durante 24 años con el objetivo de investigar su mortalidad y riesgo de cáncer. Se encontró que la mortalidad, era más alta en los portadores diagnosticados antes de sus 15 años, la proporción de mortalidad estandarizada fue 2.0 y fue calculada a través de una comparación entre los eventos observados en el estudio y las tasas de la población. Las causas principales de muerte fueron enfermedades del sistema nervioso y anormalidades congénitas, por lo que eran portadores desbalanceados que si generaron un fenotipo clínico. Adicionalmente, se encontró que el $59.4 \%$ de individuos eran mujeres. Representaban, el $63 \%$ de los individuos diagnosticados entre los 15 y 44 años. Por último, las translocaciones robertsonianas que involucran a los cromosomas 13 y 14 fueron mayoría con un $62.8 \%$ (41).

En China, se realizó una revisión de 872 translocaciones robertsonianas identificadas en un laboratorio de diagnóstico y se encontraron 583 balanceadas, 246 desbalanceadas, 9 mosaicos y 18 complejas. El $93 \%$ de las translocaciones balanceadas eran adultos (544) con infertilidad, abortos espontáneos o descendencia con anormalidades cromosómicas. Las translocaciones $\operatorname{der}(13 ; 14)$ y $\operatorname{der}(14 ; 21)$ constituían el $59.4 \%$ y $14.7 \%$ respectivamente. De estos, 227 hombres (41.73\%) y 317 mujeres (58.27\%), mostraron diferencia significativa entre los grupos. E1 98\% de las translocaciones desbalanceadas eran pacientes pediátricos y casi todas involucraban al cromosoma 21 (42).

Un estudio de cohorte se realizó en Sri Lanka, con el objetivo de reportar la frecuencia y espectro de translocaciones cromosómicas. De un total de 15,684 individuos, se encontró que 277 (1.7
\%) tenían translocación. De ellos, 160 fueron portadores de translocación robertsoniana, 145 individuos eran menores de 18 años y dentro de estos $142(97.9 \%)$ poseen translocación desbalanceada. Ciento treinta y ocho fueron referidos por una anormalidad congénita, retraso en el desarrollo o discapacidad intelectual, que incluían a 91 con síndrome de Down. Todos los individuos mayores de 18 años (132) tenían translocaciones balanceadas (90 mujeres, lo que las hizo mayoria con $68.2 \%$ ), 84 de ellos han tenido problemas de fertilidad y 48 han tenido descendencia con anormalidades congénitas. Los cromosomas 21, 14 y 13 fueron los más involucrados en las translocaciones robertsonianas; 72 individuos (45\%) portaban der (14q;21q), 30 (18.7\%) portaban der(21q;21q) y 34 (21.25\%) portaban der(13q;14q) (43).

Con los datos reportados se pueden señalar las siguientes observaciones: en los estudios realizados en Sri Lanka (42), China (43) y Gran Bretaña (41), los portadores sanos de translocaciones balanceadas y robertsonianas eran adultos por amplia mayoría dentro de estos grupos. Como es de esperar gran parte de estos individuos tenían problemas de fertilidad o descendencia con malformaciones congénitas. Además se observa mayoría de mujeres (58.7\% en el estudio chino, $68.18 \%$ en el de Sri Lanka y $63 \%$ en el británico). La mayor diferencia entre estos grupos fue la translocación robertsoniana más común, en el estudio chino la que involucra los cromosomas 13 y 14 ocupó el $59 \%$ y $62.8 \%$ en el británico. A diferencia de Sri Lanka en donde a translocación 14/21 fue la más frecuente (45\%).

Por otro lado, en Egipto se realizó un estudio estadístico enfocado en parejas que habían sufrido al menos dos abortos espontáneos recurrentes. De 224 parejas, se encontró que 28 individuos tenían una anormalidad cromosómica, cuatro de ellos $(0.89 \%)$ con una translocación robertsoniana (44). En Monterrey, México con el objetivo de estimar la prevalencia de anormalidades cromosómicas y variantes poliformes en 158 parejas con historial de abortos espontáneos repetidos, se realizaron estudios cromosómicos y se encontró translocación robertsoniana en dos $(1.27 \%)$, además se observó 


\section{Esquivel-Quezada et al}

que la prevalencia de anormalidades cromosómicas en mujeres dividida entre la prevalencia en hombres resulta en una razón de 1.4:1 (45). En Guadalajara, México también, al determinar la prevalencia de anormalidades cromosómicas se estudiaron 938 parejas con desordenes reproductivos. De estas parejas 542 tuvieron abortos espontáneos recurrentes, 356 descendencia con malformaciones y 41 esterilidad. Se encontraron 10 parejas portadoras de translocación robertsoniana (1.06\%). En total se encontró a 52 parejas con una aberración cromosómica, de estas en 28 (53.8\%) la mujer era portadora y en $24(46.2 \%)$ el hombre, se obtiene una razón de 2.3:1 respectivamente (46).

En el oeste de la India se hizo un estudio para caracterizar la prevalencia y tipos de anormalidades cromosómicas en 1,728 muestras prenatales de alto riesgo, se detectó cariotipo anormal en 125 (7.2\%), la trisomía 21 en $46(2.7 \%$ ) y fue la anormalidad más común, seguida por la trisomía 18 en 11 muestras $(0.6 \%)$ y trisomía 13 en dos $(0.1 \%)$. Se detectaron translocaciones robertsonianas en 20 muestras que corresponden al $1.2 \%$ (47).

Las poblaciones estudiadas en Egipto (44), los dos estudios mexicanos $(45,46)$ y el hindú (47) comparten la característica de poseer una translocación robertsoniana, al tratarse de parejas con historial de abortos espontáneos repetidos o descendencia con malformaciones y de muestras prenatales de alto riesgo. Se observa que las translocaciones robertsonianas constituyen el 0.89 $\%, 1.27 \%, 1.06 \%$ y $1.2 \%$ respectivamente en estas poblaciones. El promedio de estos porcentajes es $1.10 \%$. Si lo contrastamos con que la frecuencia en la población general de las translocaciones robertsonianas mencionada anteriormente es de 1 en cada 1000 recién nacidos $(0.1 \%)(4)$, se observa que es 11 veces más probable encontrar individuos con una translocación robertsoniana en grupos con antecedentes de abortos espontáneos o descendencia con malformaciones congénitas.

\section{CONCLUSIONES}

Las translocaciones robertsonianas son un evento genético aleatorio del cual existen pocos esfuerzos para lograr su detección, debido a que gran parte son portadores fenotípicamente normales. El indicativo más fuerte para sospechar de una translocación robertsoniana en un individuo y su familia es un historial de abortos espontáneos recurrentes o la presencia de descendencia con malformaciones congénitas La integración y análisis de los informes en el periodo de que se reporta, mostraron que la probabilidad de encontrar individuos con una translocación robertsoniana es 11 veces mayor en grupos con antecedentes de abortos espontáneos o descendencia con malformaciones congénitas, comparados con la población general. Este trabajo presenta la actualización de la información, facilita la comprensión del tema y contribuye a la difusión en personal médico de primer contacto y estudiantes o docentes del área de la salud.

\section{REFERENCIAS}

1. Torriani GP, González N, Bravo Y, Maceira L, Tejeda A. Reordenamientos cromosómicos estructurales balanceados en un caso de diagnóstico prenatal citogenético. Rev Cubana Genet Comunit. 2014 Mar; $8(3), \quad 28-31$. https:/www.medigraphic.com/pdfs/ revcubgencom/cgc-2014/cgc143f.pdf.

2. Díaz-Véliz PA, Vidal-Hernández B, Pérez-Pérez A, Velázquez-Martínez T. Detección de translocaciones cromosómicas mediante diagnóstico citogenético. Cienfuegos, 2006-2016. Medisur. 2018 Feb; 16(1); 29-34. https:/www.redalyc.org/articulo.oa?id=180061480006.

3. Rodríguez-Salvador A. Valoración del diagnóstico clínico de los factores genéticos implicados en la infertilidad masculina. Trabajo fin de master. Hospital Universitario de Cruces. Universidad de Cantabria. Cantabria. España. Octubre 2018. https://repositorio.unican.es/xmlui/ bitstream/handle/10902/14955/Rodr\%C3\%ADguez\%20 Salvador $\% 2 c \% 20$ Alba.pdf?sequence $=1 \&$ is Allowed $=y$.

4. Guapi VH, Jiménez GF, Mera SP. Translocación rob(13; 15) (q10; q10): a propósito de un caso. Univ Me. 2018 Jun; 59(2). doi: https://doi.org/10.11144/Javeriana. umed59-2.tran.

5. Barrios MA, Méndez LA. Enfoque de los principales factores causales en los trastornos reproductivos. Rev Cubana Obstet Ginecol. 2014 Jun; 40(2): 246-57. http:// scielo.sld.cu/scielo.php?script $=$ sci_arttext\&pid=S0138600X2014000200011.

6. Kaddouri S, Blanes R, Concepción C, Hess S, González $\mathrm{J}$, Vaca $\mathrm{R}$, et al. Los varones portadores de una translocación cromosómica presentan es-permiogramas 
más patológicos que los varones normales XY. Rev Ibeo Fer. 2018 May; 35(4). https://bit.ly/2Dpaczy.

7. Lalonde E, Stefan R, Lin F, Dulik M, Skraban CM, Spinner NB. Genomic Diagnosis for Pediatric Disorders: Revolution and Evolution. Front Pediatr. 2020 Jul; 8(373). doi: 10.3389/fped.2020.00373

8. Ewa W, Marta O, Nataliya H, Vyacheslav B, ChernykhMaciej K. The effect of robertsonian translocations on the intranuclear positioning of Nors (nucleolar organizing regions) in human sperm cells. Sci Rep. 2019 Feb; 9(2213). doi: https://doi.org/10.1038/s41598-01938478-x.

9. Godo A, Blanco J, Vidal F, Sandalinas M, Garcia-Guixé E, Anton E. Altered segregation pattern and numerical chromosome abnormalities interrelate in spermatozoa from Robertsonian translocation carriers. Reprod Biomed Online. 2015 Jul; 31(1): 79-88. doi: 10.1016/j. rbmo.2015.04.003.

10. Beyer CE, Willats E. Natural selection between day 3 and day 5/6 PGD embryos in couples with reciprocal or Robertsonian translocations. J Assist Reprod Genet. 2017 Nov; 34(11): 1483-92. doi: 10.1007/s10815-0171009-0.

11. Zhang L, Jiang W, Zhu Y, Chen H, Yan J, Chen ZJ. Effects of a carrier's sex and age on the segregation patterns of the trivalent of Robertsonian translocations. J Assist Reprod Genet. 2019 Sep; 36(9): 1963-9. doi: 10.1007/s10815-019-01534-6.

12. Paez-Bermeo MC. Alteraciones cromosómicas como factor etiológico en el aborto espontáneo y sus complicaciones más frecuentes. Examen Complexivo. Unidad Académica de Ciencias de la Salud. Universidad de Machala. Machala. Ecuador. Noviembre 2017. http://186.3.32.121/bitstream/48000/11883/1/PAEZ\%20 BERMEO\%20MARY\%20CARMEN.pdf.

13. Castillo-Taucher S, Fuentes-Soto AM, PaulosMillanao A, Rosa-Rebaza E. Estudio cromosómico en abortos espontáneos. Rev Chil Obstet Ginecol. 2014; 79(1): 40-6. doi: https://dx.doi.org/10.4067/S071775262014000100007.

14. Kar B, Sivamani S, Kundavi S, Varma TR. The importance of cytogenetics and associated molecular techniques in the management of patients carrying robertsonian translocation and their pregnancy outcome by intracytoplasmic sperm injection. J Obstet Gynaecol India. 2018 Apr; 68(2): 93-7. doi: 10.1007/s13224-0170999-y.

15. Ozen F, Saglam Z, Kurtulgan H, Ozen A. A balanced robertsonian translocation $(13 ; 14)$ of a male: a case report. J Med Cases. 2014; 5(12): 615-7 https://www. journalmc.org/index.php/JMC/article/view/1921/1357.

16. Vikraman SN, Chandra V, Balakrishanan B, Batra M, Kuriakose R, Kannoly G. A rare balanced parental $t$ (21q; 21q) robertsonian translocation that results in
Down syndrome in all viable pregnancies. Int $J$ Reprod Contracept Obstet Gynecol. 2015; 4(2): 514-7. doi: 10.5455/2320-1770.ijrcog20150451.

17. Alhalabi N, Al-Achkar W, Wafa A, Kenj M, Alhalabi M. De novo balanced robertsonian translocation $\operatorname{rob}(22 ; 22)$ (q10;q10) in a woman with recurrent pregnancy loss: A Rare Case. J Reprod Infertil. 2018 Jan-Mar; 19(1): 61-6. PMID: 29850449.

18. Ahmadi-Shadmehri A, Tavakkoly-Bazzaz J, Darbouy M, Tabatabaiefar MA. A 15;15 translocation in a couple with repeated abortions: case report. RABMS. 2020; 6(1): 14-7 http://ijrabms.umsu.ac.ir/article-1-92-en.html.

19. Poornima S, Daram S, Krishna R, Hasan Q. Robertsonian and balanced reciprocal translocation in both child and mother with a history of recurrent abortions. $J$ Reprod Infertil. 2020 Jan-Mar; 21(1): 65-7. PMID: 32175267.

20. Kusre G, Sarma M, Nirmolia T, Shankarishan P. Robertsonian translocation $\mathrm{t}(21 ; 21)$ in a female born to normal parents: a case report. J Clin Diagn Res. 2015 Jan; 9(1): GD01-2. doi: 10.7860/JCDR/2015/10638.5372.

21. Kolgeci S, Kolgeci J, Azemi M, Daka A, Shala-Beqiraj $\mathrm{R}$, Kurtishi I, et al. Dermatoglyphics and reproductive risk in a family with robertsonian translocation 14q;21q. Acta Inform Med. 2015 Jun; 23(3): 178-83. doi: 10.5455/ aim.2015.23.179-183.

22. Jaiswal S, Upadhyay A, Ali A, Upadhyay S, Kumar A, Rai A. Two familial cases of robertsonian translocations 13; 14 and its clinical consequences. J Genet Syndr Gene Ther. 2016 Jan; 7(1): 1-4. doi:10.4172/21577412.1000283.

23. Cho CH, Shin JH, Nam MH, Lim CS, Lee CK, Cho $\mathrm{Y}$, et al. A case report of an infant with robertsonian translocation $(15 ; 22)(\mathrm{q} 10 ; \mathrm{q} 10)$ and literature review. Ann Clin Lab Sci. 2016 Winter; 46(1): 102-5. PMID: 26927352.

24. Israni A, Mandal A. De novo robertsonian translocation $\mathrm{t}(21 ; 21)$ in a child with Down syndrome. $J$ Nepal Paediatr Soc. 2017 Oct; 37(1): 92-4: doi: https://doi. org/10.3126/jnps.v37i1.16184.

25. Rachisan AL, Niculae AS, Tintea I, Pop B, Militaru $\mathrm{M}$, Bizo A, et al. Association of fragile $\mathrm{X}$ syndrome, robertsonian translocation $(13,22)$ and autism in a child. Clujul Med. 2017; 90(4): 445-8. doi: 10.15386/ cjmed-763.

26. Malekpour N, Kormi SMA, Azadbakht M, Yousefi M, Hasanzadeh-Nazar Abadi M. The survey of double robertsonian translocation $13 \mathrm{q} ; 14 \mathrm{q}$ in the pedigree of 44; XX woman: a case report. Int J Mol Cell Med. 2017 Fall; 6(4): 243-8. doi: 10.22088/BUMS.6.4.243.

27. Sha J, Liu F, Zhang B, Huang Y, Zhang Q, Juan G, et al. Next-generation sequencing and karyotype analysis for the diagnosis of robertsonian translocation type trisomy 13: a case report. Iran J Public Health. 2017 Jun; 46(6): 848-51. PMID: 28828329. 
28. Nikfar A, Mansouri M, Abhari GF. A rare de novo robertsonian translocation $\mathrm{t}(21 \mathrm{q} ; 21 \mathrm{q})$ in an iranian child with down syndrome: a case report. Acta Med Iran. 2019 Feb; 57(8): 522-4. doi: https://doi.org/10.18502/acta. v57i8.2430.

29. Gumus E. Two rare variants of Down syndrome: DownTurner syndrome and Down syndrome with translocation (13;14): a case report. Iran J Public Health. 2019 Nov; 48(11): 2079-82. PMID: 31970109.

30. Farzaneh MR, Khavari M, Hasanpour M. Baghmollaei EM. Simultaneous occurrence of Turner syndrome and robertsonian translocation in a girl with short stature: a case report. Iran South Med J. 2019 Jul; 22(3): 184-90. doi: $10.29252 /$ ismj.22.3.184.

31. Xu HH, Dai MZ, Wang K, Zhang Y, Pan FY, Shi WW. A rare Down syndrome foetus with de novo 21q;21q rearrangements causing false negative results in noninvasive prenatal testing: a case report. $B M C \mathrm{Med}$ Genomics. 2020 Jul 6;13(1): 96. doi: 10.1186/s12920020-00751-8.

32. Wang X, Pang H, Shah BA, Gu H, Zhang L, Wang H. A male case of Kagami-Ogata syndrome caused by paternal unipaternal disomy 14 as a result of a robertsonian translocation. Front Pediatr. 2020 Mar; 8(88). doi: 10.3389/fped.2020.00088.

33. Saikia D, Kusre G. Evidence of rare robertsonian translocation $(14 ; 21)$ inherited from mother: a case report from North-Eastern state Assam. Int J Health Sci Res. 2016; 6(11): 278-81. https://www.ijhsr.org/IJHSR_ Vol.6_Issue.11_Nov2016/43.pdf.

34. Plaiasu V. Down syndrome - genetics and cardiogenetics. Maedica (Bucur). 2017 Sep; 12(3): 208-13. PMID: 29218069.

35. Flores-Ramírez F, Palacios-Guerrero C, García-Delgado C, Morales-Jiménez AB, Arias-Villegas CM, Cervantes A, et al. Cytogenetic profile in 1,921 cases of trisomy 21 syndrome. Arch Med Res. 2015 Aug; 46(6): 484-9. doi: 10.1016/j.arcmed.2015.08.001.

36. Xie Y, Xu Y, Wang J, Miao B, Zeng Y, Ding C, et al. Preliminary analysis of numerical chromosome abnormalities in reciprocal and Robertsonian translocation preimplantation genetic diagnosis cases with 24-chromosomal analysis with an aCGH/SNP microarray. J Assist Reprod Genet. 2018 Jan; 35(1): 17786. doi: 10.1007/s10815-017-1045-9.

37. Yan J, Zhang Q, Chen Y, Chen ZJ. Birth of a mosaic nonDown syndrome offspring to a $45, \mathrm{XY}, \mathrm{t}(21 ; 21)(\mathrm{q} 10 ; \mathrm{q} 10)$ homologous robertsonian translocation carrier: a case report. J Reprod Med. 2017 May-Jun; 62(5-6): 337-41. https://europepmc.org/article/med/30028100.
38. Xu J, Zhang Z, Niu W, Yang Q, Yao G, Shi S, et al. Mapping allele with resolved carrier status of Robertsonian and reciprocal translocation in human preimplantation embryos. Proc Natl Acad Sci U S A. 2017 Oct 10; 114(41): E8695-E702. doi: 10.1073/ pnas. 1715053114.

39. Zhou Z, Ma YL, Li Q, Zhang Y, Huang YH, Tu ZH, et al. Clinical application of oligo array-CGH for detecting balanced translocations in preimplantation genetic diagnosis. Int J Clin Exp Pathol. 2017 Jul; 10(7): 782135. PMID: 31966630.

40. Lukaszuk K, Pukszta S, Ochman K, Cybulska C, Liss J, Pastuszek E, et al. Healthy baby born to a robertsonian translocation carrier following next-generation sequencing-based preimplantation genetic diagnosis: a case report. AJP Rep. 2015 Oct; 5(2): e172-5. doi: $10.1055 / \mathrm{s}-0035-1558402$.

41. Schoemaker MJ, Jones ME, Higgins CD, Wright AF; United Kingdom Clinical Cytogenetics Group, Swerdlow AJ. Mortality and cancer incidence in carriers of balanced robertsonian translocations: a national cohort study. Am J Epidemiol. 2019 Mar; 188(3): 500-8. doi: 10.1093/aje/kwy266.

42. Zhao WW, Wu M, Chen F, Jiang S, Su H, Liang J, et al. Robertsonian translocations: an overview of 872 Robertsonian translocations identified in a diagnostic laboratory in China. PLoS One. 2015 May; 10(5): e0122647. doi: 10.1371/journal.pone.0122647.

43. Paththinige CS, Sirisena ND, Kariyawasam U, Dissanayake V. The frequency and spectrum of chromosomal translocations in a cohort of sri lankans. BioMed Research International. 2019. https://doi. org/10.1155/2019/9797104.

44. Elhady GM, Kholeif S, Nazmy N. Chromosomal aberrations in 224 couples with recurrent pregnancy loss. J Hum Reprod Sci. 2020 Oct-Dec; 13(4): 340-8. doi: 10.4103/jhrs.JHRS_11_20.

45. De la Fuente-Cortés BE, Cerda-Flores RM, DávilaRodríguez MI, García-Vielma C, De la Rosa Alvarado RM, Cortés-Gutiérrez EI. Chromosomal abnormalities and polymorphic variants in couples with repeated miscarriage in Mexico. Reprod Biomed Online. 2009 Apr;18(4):543-8. doi: 10.1016/s1472-6483(10)60132-0.

46. Meza-Espinoza JP, Anguiano LO, Rivera $\mathrm{H}$. Chromosomal abnormalities in couples with reproductive disorders. Gynecol Obstet Invest. 2008 Jul;66(4):237-40. doi: 10.1159/000147170.

47. Sheth F, Rahman M, Liehr T, Desai M, Patel B, Modi $\mathrm{C}$, et al. Prenatal screening of cytogenetic anomalies a Western Indian experience. BMC Pregnancy Childbirth. 2015 Apr; 15(90). doi: 10.1186/s12884-015-0519-y. 